\title{
Phased Antenna Array Implementation with USRP
}

\author{
Markku Jokinen \\ Centre for Wireless Communications \\ University of Oulu \\ Erkki Koiso-Kanttilan katu 3 \\ 90570 Oulu, Finland \\ Email: markku.jokinen@oulu.fi
}

\author{
Marko Sonkki \\ Centre for Wireless Communications \\ University of Oulu \\ Erkki Koiso-Kanttilan katu 3 \\ 90570 Oulu, Finland \\ Email: marko.sonkki@oulu.fi
}

\author{
Erkki Salonen \\ Centre for Wireless Communications \\ University of Oulu \\ Erkki Koiso-Kanttilan katu 3 \\ 90570 Oulu, Finland \\ Email: erkki.salonen@oulu.fi
}

\begin{abstract}
This paper presents the implementation of a phased antenna array with Universal Software Radio Peripheral (USRP). The antenna elements of the array are driven by individual USRP platforms. Hence, the synchronization and calibration of different platforms are required in phase and amplitude. Calibration issues are discussed including limitations of calibrating USRPs which use WBX radio card. Planar four-element linear antenna array was used in the measurements. The prototype antenna array driven by USRPs was measured in an anechoic chamber and results show that it can create directional radiation pattern in the receiver and transmitter directions. Results show also that radiation pattern can be steered in the desired direction, by changing the excitation phase of different antenna ports.
\end{abstract}

\section{INTRODUCTION}

Smart antennas are used to increase a signal to noise ratio of a communication system, by pointing the radiated energy in a direction of the receiver or receiving energy from the direction of the transmitter. The simplest smart antenna implementation is an uniform linear array (ULA) [1], where similar antennas are placed along a line with constant separation between adjacent antenna elements. All the antenna inputs need to be driven with known phase and amplitude value. This requires phase and amplitude synchronised antenna drivers. The practical implementation of a phased antenna array with software defined radio (SDR) platform is studied in this paper. Although, the theory of phased arrays is very well known, the practical implementation of such system has many challenges and design procedures are not available.

By using smart antennas, the spatial efficiency of the data transmission system can be improved. This is because energy can be directed to the desired direction and distortion signals can be mitigated. This can be seen as one way of increasing a spatial reuse of the system, because smart antennas allow multiple devices to operate closer to each other in the same time and frequency resources [2]. One way of enhancing spatial efficiency is so called spectrum sharing, where two separate systems are using the same spectral resources, but are separated by at least minimum distance apart which ensures interference free operation of the both systems [3]. With smart antennas the distance between two systems can be reduced. In general there are also other options to achieve efficiency. Multiple input multiple output (MIMO) technique can be used to increase the throughput of the data link, increasing the reliability of the data link or to service multiple users with the same time and frequency resources using multiple antenna elements. A disadvantage of the MIMO technique is its requirement of full channel state information, so the processing power requirement can be exhausting, especially when using SDR platforms. With the smart antenna system only angle of arrival information is required, which is computationally much easier, that is why we selected to implement a smart antenna system.

Smart antennas can be used in multiple ways. It can be used to create steerable radiation patterns, which can direct radiated energy in the desired direction. This is beneficial when the received signal level is otherwise low and in the case when the transmitted signal is desired to be undetected by other users. In satellite communications for example, signal levels are typically very low due to the long distances and limited transmission powers, that is why directional antennas are needed. If the receiver platform is moving, then direction of the reception needs to be constantly adjusted. In contrast, typically in military applications signals need to be kept hidden from the opposing side. The network topology is constantly changing, because of the mobility of the troops, which also requires tracking of angle of arrival. In both, military scenario and in commercial communications it might be desirable to suppress signals in some directions on top of steering the direction of maximum radiation. It will increase the signal to interference ratio, hence to make the system more resistant to intentional interference or interference caused by dense network deployment. This can be achieved with the smart antenna system.

In this paper, we concentrate on the implementing ULA with Universal Software Radio Peripheral (USRP) SDR platform. Creating an antenna array with USRP platform requires that all antenna ports are synchronised in the same phase and amplitude. Work presented in this paper is first step in creating a test environment, which can utilize transmitted and receiving radiation pattern to increase signal quality. Later this system will be extended with the capability of producing various channel conditions in over-the-air (OTA) environment. Hence, the system can be thoroughly tested. With the used SDR platform also wide range of spectral resources enabled by wideband antennas can be utilized. 


\section{Antenna ARray Theory}

To increase the directivity of the antenna system, the electrical dimensions of the antenna need to be increased. This can be made by increasing the physical dimensions of the antenna element. Another option is to use multiple antenna elements as an antenna array. The array of elements is usually formed with identical radiation elements, but is not necessity as it just makes analysis of the array more practical. The individual elements can be in any form such as, wire, aperture or patch. The simplest antenna array is an uniform linear array (ULA) [1]. The total field of the antenna array is a vector addition of the antenna fields of individual antennas. This assumes that current distribution of each element is identical in comparison with the isolated element. In practice, however this is not the case, because there is mutual coupling between elements.

An array of antennas is used to create constructive and destructive regions in different directions around the antenna. In the case of identical antenna elements, the total array radiation pattern can be calculated by multiplying single element radiation pattern with an array factor (AF) [1]. AF is depending on the array geometry, the number of elements and an excitation phase of an individual antenna. An N-element linear array with progressive phase shift, $\beta$, between elements, have AF of

$$
\mathrm{AF}=\sum_{n=1}^{N} e^{j(n-1) \psi},
$$

with

$$
\psi=k d \cos \theta+\beta,
$$

where $\theta$ is the angle of interest, $k=2 \pi / \lambda$, where $\lambda$ is the wave length, $\mathrm{d}$ is the distance between antenna elements and $\mathrm{N}$ is the number of antenna elements. If radiating elements are isotropic point sources, AF equals the total array radiation pattern of the antenna constellation. The array factor can also be presented after simple mathematical manipulation and normalization to unity as

$$
\mathrm{AF}=\frac{1}{N}\left[\frac{\sin \left(\frac{N}{2} \psi\right)}{\sin \left(\frac{1}{2} \psi\right)}\right] .
$$

In order to limit the number of radiation maximum to one, the separation between antenna must fulfill $d<\lambda$. If $d=\lambda$ is allowed, the broadside radiation maximum will form additional maximum also along the axis of the array. These additional maximums are called grating lopes. Number of nulls depends on separation between elements and a phase excitation of the antennas [1].

The direction of the maximum of the antenna array is the function of progressive phase shift, $\beta$, so by changing $\beta$, the direction of radiation maximum can be chanced. The excitation phase to create a maximum in direction $\theta_{0}$ can be calculated with

$$
\psi=k d \cos \theta_{0}+\beta=0 \Rightarrow \beta=-k d \cos \theta_{0} .
$$

Hence, by modifying the progressive phase of each antenna angle of the array, the maximum can be controlled. This allows realtime antenna pattern configuration when the phase of individual antenna port is controlled.

\section{Antenna ARray}

The planar four-element linear antenna array presented in [4] was used in this work. In this design, four similar planar microstripline-fed open-ended quarter-wave slot antenna elements are arranged as an array on the same substrate. The prototype antenna array is presented in Figure 1. The antenna array used in this paper consist of a wideband radiating elements operating from $2 \mathrm{GHz}$ to $6 \mathrm{GHz}$. The feed of a single element is designed to excite the first order of the fundamental radiating mode. The element spacing is kept as narrow as possible to still achieve low mutual coupling between the elements. To avoid creating grating lobes, element spacing smaller than $0.8 \lambda$ should be used. The antenna element spacing of the prototype array is $44.8 \mathrm{~mm}$, which is $0.33 \lambda$ with 2.2 $\mathrm{GHz}$ center frequency used in this work. The radiated field of the array is linearly polarized. The array was preliminary designed for the wireless open-access research platform (WARP) [5] with beam steering capability [6]. Because WARP has some challenges in creating transmit beamforming, the platform was chanced to USRP for this work.

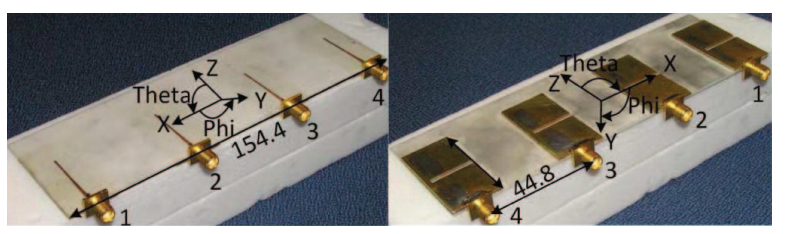

Fig. 1. Prototype antenna with dimensions (in millimeters) and coordinate system [4].

\section{USRP \& IMPLEMENTATION}

Universal Software Radio Peripheral (USRP) is a software defined radio (SDR) platform which is capable of converting RF signal into digital baseband IQ-samples or vice versa. USRP is used with a host computer through a high-speed data link. Baseband digital signal is transmitted to/from a host computer with open source USRP hardware driver (UHD). This makes the platform very flexible in implementing different software defined radio systems, with user selected software solution.

UHD is supported by multiple signal processing software, like GNU Radio, LabVIEW and Matlab. GNU Radio [7] is a free tool for creating SDR solutions, it is the most common software used with USRP. National Instruments offers LabVIEW support for USRP with NI-USRP drivers. Matlab has its own hardware support baggage, which is able to communicate with USRP. We chose the GNU Radio, because of its widest support for synchronising multiple platforms and because it is freely available.

There are many ways to connect host computer and USRP depending on the series used. Network series USRPs are connected with Gigabit Ethernet interface to a host computer. 
The bus series on the other hand is connected with USB interface. In addition, embedded series offers a stand alone solution which does not require a separate host computer. In this work, the USRP N210 platform [8] was used, because it is capable of external clock synchronisation, which is essential when creating a phased antenna array. Also, network series offers a higher dynamic range and bandwidth compared to the bus series.

The daughter card is a modular radio interface of USRP. We used the WBX daughter card as on a radio interface, which is capable of $50 \mathrm{MHz}$ to $2,2 \mathrm{GHz}$ center frequency with 100 $\mathrm{mW}$ output power.

For synchronizing an array of USRPs, an OctoClock-G timing reference and distribution system [9] was used. A diagram of all necessary connections in the system is presented in Figure 2. The figure illustrates also data connections and IP addresses of different devices.

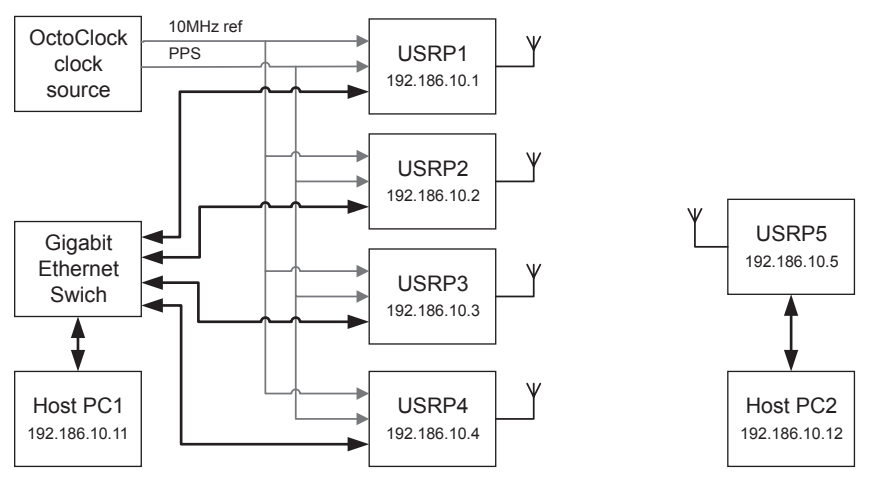

Fig. 2. Connection diagram.

\section{A. Calibration}

In this work, a driver for the four element ULA array, presented in Section III, was created. The array can act as a transmitter or as a receiver. The other end uses only one antenna. ULA radiation pattern can be controlled by changing the excitation phase or amplitude of the individual elements. This requires that all antenna drivers need to be synchronized all the way to the antenna port in frequency, phase, time and amplitude. With SDR platforms, this requires calibration and an external reference clock source to be used which is common for all antenna drivers. Phase and frequency synchronisation requires common $10 \mathrm{MHz}$ clock signal for all antenna drivers and calibration of the ports. Time synchronisation requires a separate external pulse per second (PPS) signal to be used [10]. The amplitude of antenna ports is calibrated by using one port as a reference.

Even though all USRPs are sharing the same external clock and PPS timing signal, there can be still phase difference between antenna ports due to the different length in cabling both in RF signal between board and antenna and in clock signals from clock source to board. That is why we need to calibrate the receiver and the transmitter side of the antenna array. The antenna array itself is reciprocal, but in USRP the transmit and receive chains are separated, so they have different phase and amplitude variations. Hence, we need to calibrate $\mathrm{Rx}$ and $\mathrm{Tx}$ paths separately. Calibration is done by connecting the antenna ports of USRPs with power splitter/combiner. By doing this, we replace antennas and propagation medium from Figure 2 with known and stable transmission line. Now we can transmit periodic pulses of sine wave from the single antenna port (USRP5) and receive the signal with other USRPs. Pulse like calibration signal is needed, because the start and the end of the pulse will define the phase of the sine wave in the time domain. This is also used to define the time synchronization of USRPs. With an ideal configuration all phases should be in sync. In reality they are not, thus we introduce phase shift to all antenna array driving USRPs $(1-4)$, which will compensate phase differences between different platforms. Phases are aligned visually in the receiver, where all four received signals can be shown in the same plot. After the receiver antenna array is calibrated, the same calibration needs to be performed for the transmitter side. In this case array driving USRPs transmit the same phase sine wave from all antennas and we receive those with USRP5. For the transmitter array an appropriate phase shift is introduced so that the received signals have the same phase. This time receiver is naturally summing up all received signals, and aligning them requires turning on individual elements one by one.

Together with phase calibration, also signal amplitude levels were calibrated. This was done with USRP power adjustment, in which the receiver amplifier level can be adjusted with $0.5 \mathrm{~dB}$ steps and the transmitter power amplifier with $1 \mathrm{~dB}$ steps. With this resolution, the amplitude levels were matched. Achieved power adjustment levels are presented in Table I. These values correspond to the same measured power level of all USRPs, where as differences are due to the impairments in the hardware. Differences between platforms are within $1 \mathrm{~dB}$. Adjustment values are not related to real output power level and mapping to the output power levels can be found in [11].

TABLE I

POWER ADJUSTMENT VALUES OF USRPS

\begin{tabular}{|c|c|c|c|c|}
\hline & USRP1 & USRP2 & USRP3 & USRP4 \\
\hline Rx level $(\mathrm{dBm})$ & 15.5 & 14.5 & 15 & 14.5 \\
\hline Tx level $(\mathrm{dBm})$ & 18 & 17 & 17 & 17 \\
\hline
\end{tabular}

The steering of the radiation pattern is done by introducing a progressive phase shift across the antenna array. For this we add new set of phase shifters to the design after calibration. Required phase shifts can be calculated with equation (4). These phases apply for ideal isotropic antennas, but in reality antennas are not exactly isotropic. Also the isolation between antenna elements is not ideal and other elements have an effect on the radiation pattern of individual elements. This creates differences in the amplitude of an individual element. Fortunately, the difference in amplitude has less effect on the radiation pattern than phase differences.

WBX radio card can be calibrated and used for phase accurate applications, but after re-tune of the radio or restart 
of the device there is possibility of 180 degree phase shift compared with the calibrated phase. Authors realize that better option as a radio card for building a phased antenna array would be a SBX radio card, which does not suffer on this phenomenon. Unfortunately, these were not available for this work at the time of the implementation. Therefore, the possible 180 degree offset needs to be compensated, which was done manually. Before calibration, the devices need to be switched on for several minutes, to warm up, as there is a significant drift in phase during the warming up period.

\section{Measurement Results}

The antenna array was measured in the anechoic chamber to verify that we can create directive patterns and steer the radiation maximum. The radiation pattern of the antenna array driven by USRPs was measured in over-the-air (OTA) environment. During the measurements, both, receiver array and transmitter array was measured with the same setup. A planar four-element linear antenna array [4] was used in the array side of the measurement setup and a wideband Vivaldi antenna [12] was connected to a single USRP. Used Vivaldi antenna is suitable for the used center frequency and its high directivity reduces floor reflections. Floor reflections were suppressed also with an absorption material. Polarisation of both antennas was matched. Antennas were placed 1.6 meters above the floor in the chamber and 2.2 meters apart from each other, which is clearly in far-field of the measured antenna with $2.2 \mathrm{GHz}$ center frequency. The antenna array was placed on a rotating platform, which was rotated from $-90^{\circ}$ to $90^{\circ}$, as illustrated in Figure 3, to form half of the radiation pattern. For ULA, both halves of the radiation pattern are similar and mirror images of each other.

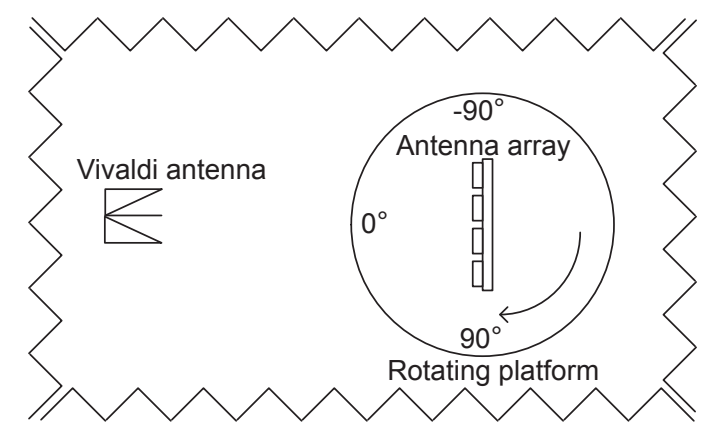

Fig. 3. Top view of the measurement setup.

A continuous sine wave was transmitted and the received power was measured with the GNU Radio QT GUI frequency sink, which gives power in $\mathrm{dBm}$. Compared with the pulse shaped calibration signal, this gives more accurate measurement results. Achieved results are presented in Figure 4 and 5 , for $0^{\circ}$ steering and $30^{\circ}$ steering respectively. Measurements were taken with $10^{\circ}$ increments, but the resolution was increased to $5^{\circ}$ close to the radiation null, to get more accurate reading of null position and depth. Results are normalised so that the maximum received power is $0 \mathrm{~dB}$. For the Rx array the measured maximum received power value was $-38.28 \mathrm{dBm}$, which is due to the link budget in the measurement setup. For the Tx array this value was $-37.31 \mathrm{dBm}$. These values are the same for both steering angles measured.

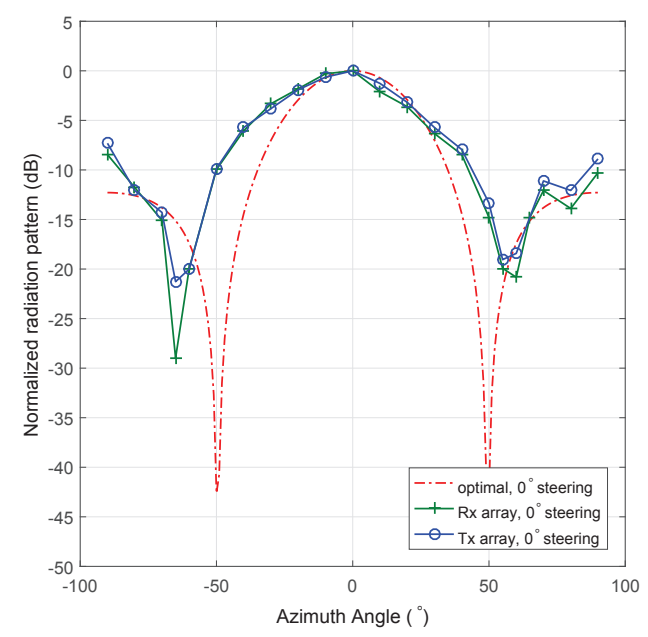

Fig. 4. Radiation pattern of $0^{\circ}$ steering for both $\mathrm{Rx}$ and $\mathrm{Tx}$ arrays compared to the optimal pattern on $2.2 \mathrm{GHz}$ carrier frequency.

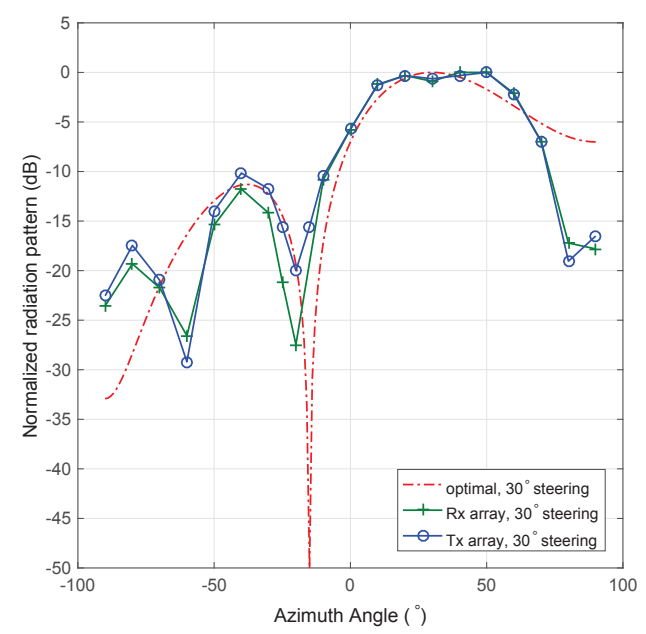

Fig. 5. Radiation pattern of $30^{\circ}$ steering for both $\mathrm{Rx}$ and $\mathrm{Tx}$ arrays compared to the optimal pattern on $2.2 \mathrm{GHz}$ carrier frequency.

It can be seen from the results, that the steering can change the angle of maximum radiation. Radiation pattern are following the basic shape of the optimal radiation pattern, which are calculated with the assumption of isotropic radiators. Measured nulls are approximately $10^{\circ}$ wider than the optimal ones, producing wider beam width. Optimal nulls are also deeper than the measured nulls. This can be caused by the radiation pattern of individual antenna elements, which are not exactly omnidirectional, but especially edge element's 
radiation patterns are more biased away from the array. Other causes of the non-ideal array pattern are inaccuracy in the calibration or in measurement setup. Rx and Tx patterns of both measurement cases are closely following each other, even though they are separately calibrated and the calibration accuracy of Tx array is not as good as Rx array. Hence, on this perspective most of the differences in the measured and optimal pattern come outside of the radios and their calibration.

\section{CONCLUSion AND Future WORK}

In this paper, phased antenna array implementation with USRP platform is presented. The antenna array was calibrated in phase and amplitude. The design was verified to be functional with measurements in anechoic chamber. Measurements show that the radiation pattern can be steered in the desired direction and the pattern roughly resembles the one calculated when assuming ideal radiators and steering.

This work creates a basic functionality of steering antenna radiation. The aim is to include it as a part of a larger test environment with MIMO-OTA [13] channel emulation. MIMOOTA can be used to create different channel environments in the anechoic chamber. USRPs will be used to steer radiation in the desired direction and suppress signals in the other directions. Effects of the controlling radiation pattern on the successful data transmission will be measured in future studies.

\section{ACKNOWLEDGMENT}

This work was supported by the Academy of Finland funded project Reconfigurable Antennas and Over-the-air Tests for Cognitive Radios (RAOTA).

\section{REFERENCES}

[1] C. A. Balanis, Antenna Theory Analysis and Design, third edition. New Jersey, United States of America: John Wiley \& Sons, Inc, 2005.

[2] I. Nicolaescu and D. Stoica, Smart antennas for wireless communications systems, 2010 Conference Proceedings ICECom, 20th International Conference on Applied Electromagnetics and Communications, 2010

[3] M. Jokinen, M. Mäkeläinen, T. Hänninen, M. Matinmikko and M. Mustonen, Minimum Separation Distance Calculations for Incumbent Protection in LSA, Cognitive Radio Oriented Wireless Networks: 11th International Conference, CROWNCOM 2016, Grenoble, France

[4] M. Sonkki, D. Pfeil, V. Hovinen and K. R. Dandekar, Wideband Planar Four-Element Linear Antenna Array, IEEE Antennas and Wireless Propagation Letters, Vol. 13, 2014.

[5] WARP Project, http://warpproject.org

[6] J. Okkonen, Uniform linear adaptive antenna array beamforming implementation with a wireless open-access research platform, Master's thesis (tech), http://urn.fi/URN:NBN:fi:oulu-201306011428, University of Oulu, Department of Computer Science and Engineering, 2013

[7] GNU Radio wiki, https://wiki.gnuradio.org/index.php/Main_Page

[8] USRP N210, https://www.ettus.com/product/details/UN210-KIT

[9] OctoClock-G CDA-2990 8-Channel Clock Distribution Module with Intergrated GPSDO, https://www.ettus.com/product/details/OctoClock-G

[10] Application Note, Synchronization and MIMO Capability with USRP Devices, Ettus Research, https://www.ettus.com/content/files/kb/mimo_ and_sync_with_usrp.pdf

[11] WBX radio Performance Data, http://files.ettus.com/performance_data/ wbx/WBX-without-UHD-corrections.pdf

[12] M. Sonkki and D. Snchez-Escuderos and V. Hovinen and E. T. Salonen and M. Ferrando-Bataller, Wideband Dual-Polarized Cross-Shaped Vivaldi Antenna, IEEE Transactions on Antennas and Propagation, Vol. 63, Issue: 6, 2015

[13] A. Khatun and V. M. Kolmonen and V. Hovinen and D. Parveg and M. Berg and K. Haneda and K. I. Nikoskinen and E. T. Salonen, Experimental Verification of a Plane-Wave Field Synthesis Technique for MIMO OTA Antenna Testing, IEEE Transactions on Antennas and Propagation, Vol. 64, Issue: 7, 2016 